\title{
Flow Field Sensing with Bio-inspired Artificial Hair Cell Arrays
}

\author{
Rodrigo Sarlo ${ }^{a}$, Joseph S Najem ${ }^{b}$ and Donald J Leo ${ }^{* c}$ \\ *Corresponding author \\ ${ }^{a}$ Mechanical Engineering Department, Virginia Polytechnic Institute and State \\ University, 445 Goodwin Hall, 635 Prices Fork Road, Blacksburg, Virginia \\ 24060, USA \\ Email: sarlo@vt.edu \\ ${ }^{b}$ College of Engineering, University of Georgia, D.W. Brooks Drive, Boyd Hall, \\ Rm 708A, Athens, Georgia 30602, USA \\ Email: jnajem@uga.edu \\ ${ }^{c}$ College of Engineering, University of Georgia, 130 Paul D. Coverdell Center, \\ Athens, Georgia 30602, USA \\ Email: donleo@uga.edu
}

\begin{abstract}
The hair cell is a biological sensor that uses microscopic hair-like structures to detect delicate motions of surrounding fluid. Inspired by this principle, an artificial hair cell (AHC) sensory method based on bio-membrane transducers is developed for airflow sensing. One-dimensional arrays built from modular AHC units measure local velocity at different points in a flow profile. Each of the AHC units uses thinly extruded glass fibers as mechanical receptors of air velocity. Hair vibrations are converted to current via hydrogel-supported (lipid bilayers) by virtue of their mechanosensitive properties. The AHC outputs are combined into one channel, requiring a demultiplexing operation to recover individual hair cell information. This is achieved by tuning each AHC hair length to a unique frequency response and recovering individual sensor information based on the frequency content of the signal. The method is entitled Tuned Frequency Response Encoding (TFRE). When several AHC units are excited simultaneously by an airflow, the resulting signal is a superposition of each sensor's individual response. The excitation at each sensor is reconstructed from the frequencies that appear in the combined output. This technique was inspired by how organisms use hair cells with tuned responses to mechanically process flow stimuli. It takes advantage of a novel AHC's high signal-to-noise ratio (compared to other membrane-based AHCs) and linear output response to flow velocity. Initial tests with linear arrays of three AHCs show success in estimating the shape of the velocity profile from an air source that varies in position and intensity. However, temporal variations in some cases in membrane size affect sensitivity properties and make accurate flow velocity estimation difficult. Nevertheless, under stable conditions, the measured velocity profiles match closely with theoretical predictions. The implementation of the array sensing method demonstrates the sensory capability of bilayer-based AHC arrays, but highlights the difficulties of achieving consistent performance with biomolecular materials.
\end{abstract}

Keywords: artificial hair cell; droplet interface bilayer; array; cell membrane; frequency response; airflow

(C) 2016. This manuscript version is made available under the Elsevier user license http://www.elsevier.com/open-access/userlicense/1.0/ 
Flow Field Sensing with Bio-inspired Artificial Hair Cell Arrays

\section{Introduction}

The term hair cell refers to biological sensors that use microscopic hair-like structures, called cilia, to detect delicate motions in surrounding fluid. Mechanoreceptive channels in these cells respond to cilia deflections by triggering neural signals [1]. Most hair cells range from tens to thousands of micrometers in size and can sense excitation energies as low as $10^{-10} \mathrm{~J}[2]$. In addition, through variations primarily in the length and stiffness of the cilia, they have adapted to detect a wide range of stimuli. Insects [3, 4], fish [5], and mammals $[1,6]$ have evolved sensory organs that utilize hair cells to sense flow, sound, vibrations and even gravity [2]. Organisms almost always employ hair cells in large numbers, synthesizing their signals to acquire sophisticated sensory information. For example, fish use rows of thousands of hair cell bundles, called lateral lines, to detect flow fields around their bodies. Blind cave fish have evolved lateral line systems to navigate obstacles in complete darkness $[7,8]$. The cochlea in mammalian ears use hundreds of linearly distributed hair cell to capture large frequency ranges. Cats, for example, can perceive frequencies in the $50-80,000 \mathrm{~Hz}$ range $[1,9,10]$. Overall, the sensitivity and versatility of hair cells applications in nature make them ideal models for small, modular and multi-purpose sensors.

The simple operational principle of hair cells has inspired several embodiments of sensors named artificial hair cells, or AHCs [11, 12]. Current AHCs seek to mimic the useful engineering properties of their biological counterparts to measure fluid movement, whether from flow or acoustic pressure waves. One branch of AHC development is aimed at practical applications at the microscopic level, exploring high aspect ratio cilia sensing elements with different materials and geometries for improved sensitivity and modularity. In order to operate at biological scales but maintain cost efficiency, established MEMS assembly techniques are used. Such designs employ a variety of sensing principles, including piezoresistive [11], capacitive [13], thermal [14], magnetic [15], piezoelectric [16], and optical [17] techniques.

Liu et al. have already developed several generations of piezoresistance based artificial hair cells for flow applications. The sensors ranged from 500 to $1100 \mu \mathrm{m}$ in cilium length and could be adapted to both air and water flow [11, 12]. The best of these sensors achieved sensitivities to water flow on the order of $0.1 \mathrm{~mm} / \mathrm{s}$ for a single cilium sensor $[12,18,19]$. Krijnen et al. used MEMS variable capacitors to transduce hair motions. The best of these sensors achieved sensitivities to airflow as low as 1 $\mathrm{mm} / \mathrm{s}$ [12]. In addition, the researchers were able to fabricate hundreds of hair cell units into rectangular arrays $[13,20]$. The arrays were mostly used to increase overall capacitance (and thus sensitivity) by connecting units in parallel. Research studies are still investigating techniques for individual unit interrogation and how to generate spatial data [21].

Overall, state of the art MEMS AHCs have demonstrated comparable size, cilium length and sensitivity to some hair cells in nature [2]. However, relatively little research has yet ventured into spacial analysis of flow fields using arrays. Chen et al. are one of the few that have attempted this task, but only for known, well defined sources. Using a lateral line inspired configuration, they were able to locate disturbance sources in water; however, their localization algorithms depended on an a priori model of the disturbance field. Furthermore, the artificial lateral line was based on hot wire anemometry and not cilia deflection [14, 22]. In general, developing effective large array signal processing methods such as flow mapping, object localization, and tracking is still an active area of research. Advancements are hindered partly by the 
75 fact that neural decoding of hair cell array information in vertebrates is not yet fully 76 understood [12].

Within the last four years, some researchers added another level of bio-inspiration to AHCs by using biomolecular transducers instead of silicon components. The goal of these sensors is to explore the engineering potential of biological materials. Work by Sarles and Leo showed that synthetic cell membranes (lipid bilayers) can be used as mechanoelectrical transducers $[23,24]$. By virtue of its mechanocapacitive properties, bending of these membranes due to hair motion induces a change in capacitance that can be measured as ionic current flow $[25,26]$. This phenomenon differs from how biological cells transduce signals, since it does not depend on mechanoreceptive channels. The first versions of these membrane-based AHCs were assembled in polyurethane substrates via the regulated attachment method or RAM [27, 28, 29]. A hydrogel "follicle" served as both a rigid support for the sensing "hair" and as a hydrophilic scaffold on which to form the lipid bilayer. Additional research has shown the possibility of creating multiple lipid bilayer networks [30, 31, 32]. Thus, a natural next step is to expand this concept to AHCs, thereby expanding applied sensing capabilities in a way that mirrors hair cell arrays in nature. Leveraging developments in lipid-on-hydrogel bilayer networks [31], the authors recently modified the AHC design into compact substrates with bilayers and hairs fully supported by movable hydrogels [33]. This foregoes the need for an external supporting structure as in the RAM technique. These AHCs were stable for at least 30 minutes and showed an order of magnitude increase in current output over previous designs. Combined with a modular design, these properties set the foundation needed to build AHC arrays. In the future, with appropriate advances in bilayer fabrication techniques, bilayer AHC arrays could be used to create organic smart materials that respond to flow, touch, sound or vibration. Such materials are useful in situations where silicon-based technology is undesirable, e.g. cochlear implants, biodegradable applications, and military stealth operations.

This study focuses on the response of membrane-based AHCs to airflow and how small linear arrays may be used to detect spatial variations in the flow magnitude. The AHC platform was developed previously by the authors for modularity and high sensitivity [33]. The paper first describes the materials and fabrication of the platform and some basic theory behind its operation. After characterizing the sensor response to airflow, it presents an array sensing strategy which involves "tuning" the lengths of individual hairs in order to map sensor excitation to unique frequencies over a single channel. The method is referred to as tuned frequency response encoding (TFRE) and is inspired by the function of the basilar membrane in the mammalian ear. The decoding algorithm uses frequency domain processing to reconstruct a spatial flow profile based on the levels of excitation at each sensor in the array. Finally, the results of the algorithm are compared to theoretical predictions and used to assess the performance of the sensing platform.

\section{Materials and methods}

\subsection{Overview of the AHC Unit}

The hair cell sensor unit consists of two main components: (1) a mechanical lever, i.e. the "hair", which deflects or vibrates when excited by a fluid flow, and (2) a transduction element, a synthetic lipid bilayer membrane, which converts the 


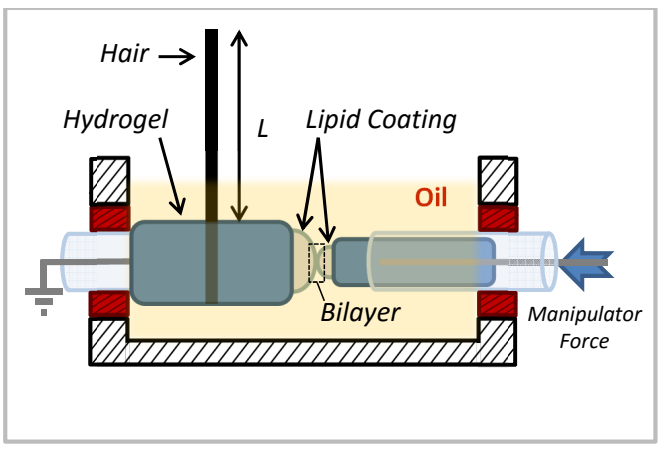

(a)

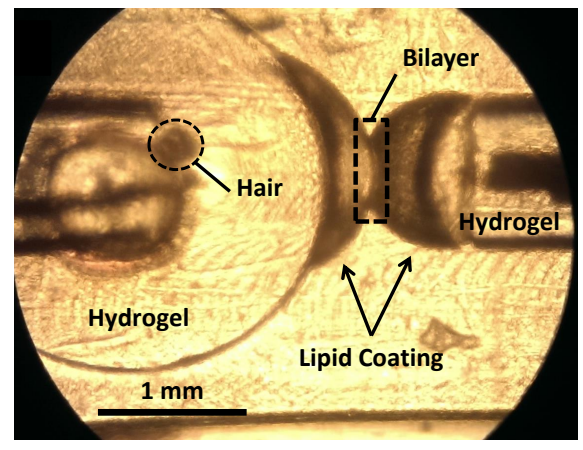

(b)

Figure 1: a) A cross-sectional view of the artificial hair cell unit and components. $L$ indicates the free length of the hair. b) A microscope image taken from underneath the substrate illustrates a typical bilayer formation.

mechanical vibrations to an electrical signal. A principal goal for the units was modularity, they should be capable of assembly into tightly-packed arrays while maintaining individual control of each system. A basic diagram of the device is shown in figure 1a. Each unit operates as a single sensor and is housed in a recessed chamber, with openings on either side for $1 \mathrm{~mm}$ diameter glass pipette tubes (1B100-4, WPI, Inc.). The tube on the left of the figure holds a "base hydrogel" which provides a rigid yet aqueous environment to anchor the lipid solution and also to support the hair. The base hydrogel had a cylindrical shape with a $3 \mathrm{~mm}$ diameter and $1.5 \mathrm{~mm}$ height. The smaller "secondary hydrogel" protrudes slightly from the right pipette and also has a lipid coating. The entire assembly is submerged in oil. The two lipid coated hydrogels are brought into contact, forming a droplet interface bilayer (DIB), shown in figure 1b. The DIB size can regulated by adjusting the position of the secondary hydrogel, thereby influencing the contact area. Silver-silver chloride $(\mathrm{Ag} / \mathrm{AgCl})$ electrodes, used for measuring the current across the bilayer, run through each pipette and insert into the hydrogels. More details on the micropipette bilayer manipulation method can be found elsewhere [34].

The substrate chamber measured $12 \times 3 \times 4 \mathrm{~mm}$ and was machined from transparent acrylic (.22 in, OPTIX), enabling microscope viewing from below. Acrylic is a hydrophobic material, which reduces the propensity of the aqueous solution to adhere to the substrate walls. The hairs were fabricated from $1 \mathrm{~mm}$ diameter solid glass rods (GR100-4, WPI, Inc.), which were heated and stretched until very thin, then cut to the desired length. The result was a slightly tapered hair with typical base diameter of about $300 \mu \mathrm{m}$ and tip diameter of about $100 \mu \mathrm{m}$.

\subsection{Droplet Interface Bilayer Components and Formation}

The DIBs in this study were formed on hydrogel supports through a hybrid of two methods developed by Bayley et al.: lipid-in DIBs [35] and lipid coated hydrogels [31]. The aqueous solution used to form lipid-in DIBs consists of $2 \mathrm{mg} / \mathrm{mL} \mathrm{1,2} \mathrm{diphytanoyl-}$ sn-glycero-3-phosphocholine (DPhPC, Avanti Polar Lipids, Inc.) phospholipids suspended in $10 \mathrm{~mL}$ of $500 \mathrm{mM}$ potassium chloride (KCl, Sigma Aldrich), $10 \mathrm{mM}$ 4-morpholinepropanesulfonic acid (MOPS, Sigma Aldrich), pH 7 ionic buffer solution. 


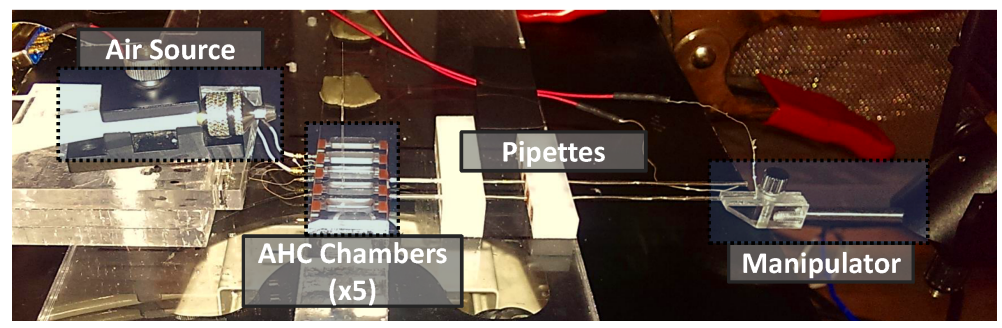

Figure 2: Experimental setup for array testing.

The preparation of this solution is described elsewhere [35]. The hydrogels are made from a mixture of $8 \mathrm{mg}$ polyethylene glycol dimethacrylate (PEG-DMA, MW $=1000$ $\mathrm{g} / \mathrm{mol}$, PolySciences, Inc.), $0.05 \mathrm{~g}$ Irgacure 2959 photoinitiator (Ciba) dissolved in $10 \mathrm{~mL}$ of the same ionic buffer solution as the phospholipids $(\sim 40 \% \mathrm{w} / \mathrm{v})$. When cured via UV light, the hydrogels photopolymerize, providing a rigid yet hydrophilic environment that serves to anchor the aqueous lipid solution. This facilitates the manipulation of aqueous droplets. During fabrication, the hydrogels were cured onto the pipettes on polyurethane molds for approximately two minutes. Each hydrogel is then inserted into the AHC chamber, submerged in hexadecane oil (99\%, Sigma), and coated with a $\sim 0.2 \mu \mathrm{L}$ lens of the aqueous lipid solution using a microsyringe. The hydrophilic-hydrophobic interface at the lens-oil boundary creates the necessary conditions for lipid monolayer self assembly, typically in 5-10 mins. The monolayer surfaces can then be brought into contact to initiate bilayer growth.

\subsection{Airflow testing of AHC Arrays}

This study focuses on arrays of three hair cells as an initial proof of concept. Figure 2 shows the experimental setup. Five AHC chambers were machined side-by-side into $0.22 \mathrm{in}$. thick acrylic with a spacing of $8 \mathrm{~mm}$. Only three of the five chambers were used in this study. The spacing is compact enough to allow the air source to excite multiple hairs at once while being separate enough for the manipulation of the pipettes. A single precision stage (SM325, WPI, Inc.) can be attached/detached to each pipette con control the membrane area. The AHCs, air source and pipette holders were attached to an acrylic platform that can be moved to allow microscope viewing of different $\mathrm{AHC}$ compartments from below.

Pressurized air was released through $3.2 \mathrm{~mm}$ diameter plastic tubing to create wide flow profile. A mass flowmeter (FMA-A2321, Omega Inc.) monitored the steadystate flow rate of the air. The flow rate was converted to velocity using equations (1) and (2) in section 3.1. The air pressure could be varied from 0-12 psi to produce flow velocities from $0-55 \mathrm{~L} / \mathrm{min}$, corresponding to maximum air speeds of 0-72 $\mathrm{m} / \mathrm{s}$. The tube diameter and flow rates for testing were chosen such that the flow had enough coverage and power to excite all three hairs at once.

Figure 3 illustrates the array testing procedure for TFRE. This section only deals with the methodology of TFRE; more details are found in section 4.2. The airsource was placed $15 \mathrm{~mm}$ vertically above the platform and $15 \mathrm{~mm}$ away from hair cells. The airflow direction was perpendicular to the bilayer membranes, as seen in the figure. This excitation direction has been experimentally shown to elicit the greatest current 


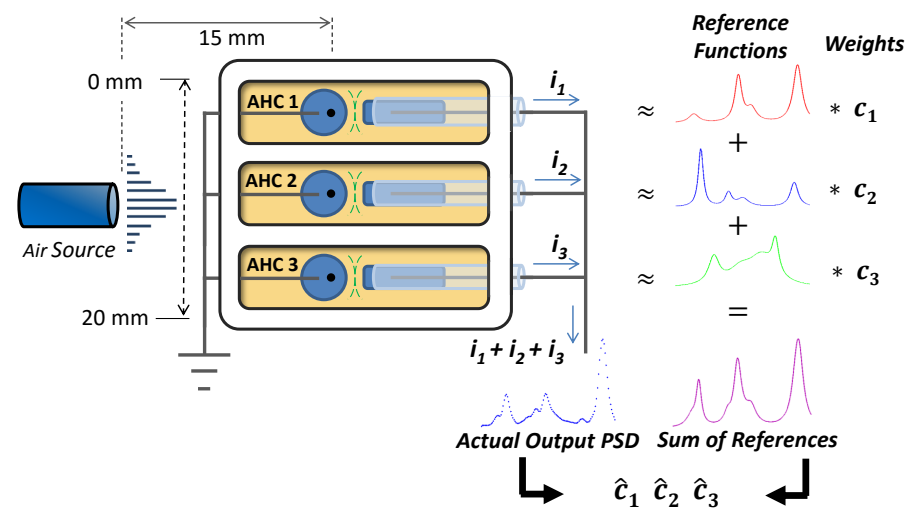

Figure 3: Illustration of an array test and TFRE. The air source is swept across the hair cells while the total output current is measured. Based on knowledge of each $\mathrm{AHC}$ frequency response (red, blue, and green plots) and a weighted fit of reference functions, the air velocity at each AHC can be estimated (see section 4.2).

response [36]. It was wide enough to excite all hairs once in varying degrees. While holding the velocity constant, the airflow was moved laterally in $2 \mathrm{~mm}$ increments for a total distance of $20 \mathrm{~mm}$. Because of the shape of the airflow profile (see section 3.1), each increment caused the air velocity felt at each AHC to change. The air source was fixed at each position approximately 5-10 seconds while the AHC output response was collected. Five of these position sweep experiments were conducted, each with the same hair lengths but different bilayers. The different hair lengths $(33 \mathrm{~mm}, 26 \mathrm{~mm}$, and $20 \mathrm{~mm}$ ) gave each AHC a unique vibrational response, as shown by the red, blue and green frequency plots on the right of figure 3. This was fundamental to the TFRE method. Further explanation on this figure is found in section 4.2, where TFRE is developed. The hair vibrations of interest were well below $1.2 \mathrm{kHz}$, thus the AHC output was sampled at $2.5 \mathrm{kHz}(1.25 \mathrm{kHz}$ nyquist frequency).

During array testing, the three AHC units were wired in a parallel configuration to an AxoPatch 200B amplifier's single measurement channel. Thus, the measured current was the combined output of the three AHCs (figure 3). The circuit was voltage-clamped at a constant bias potential of $-20 \mathrm{mV}$. The bias potential amplifies the bilayer current output (see section 3.2), but can also destabilize the bilayer. The choice of $-20 \mathrm{mV}$ struck a balance between these two factors. All measurements were conducted in a custom-made Faraday cage for shielding.

\section{Theory}

The sensory process depends on airflow exciting the hair at a particular velocity. The hair then vibrates according to this velocity and the boundary conditions imposed by the hydrogel base. Vibration of the hydrogel itself then excites the bilayer, which produces a measurable current. In this section, we introduce theoretical models to describe these interactions. This section provides an overview of the concepts needed to interpret the experimental results, namely, a description of the velocity profile produced by the air source, the hair's vibrational response to current and the bilayer 
membrane's conversion of vibration to current.

\subsection{Air Velocity Profile}

In all array experiments, the air source was a circular tube. Reynolds number calculations for the air velocities in this work $\left(10^{4}\right.$ to $\left.3 \times 10^{5}\right)$ place them in the turbulent regime. The Goertler solution for a free turbulent jet exiting a circular nozzle [37] shows the radial air velocity distribution $v(r, x)$ to be:

$$
\begin{aligned}
& v(r, x)=\frac{v_{\mathrm{m}}}{1+0.125(\sigma r / x)^{2}} \\
& v_{\mathrm{m}}=\frac{v_{0} C_{1}}{x / r_{0}}
\end{aligned}
$$

where $r$ is the radial distance from the nozzle's center, $x$ is the axial distance from the nozzle, $v_{\mathrm{m}}$ is the maximum velocity, $v_{0}$ is the uniform velocity at $x=0$ and $r_{0}$ is the nozzle radius. The constants $\sigma$ and $C_{1}$ are derived experimentally and are approximately equal to 18.5 and 5.75 , respectively [37]. The relationship in (1) describes a bell-shaped air velocity distribution with a maximum velocity occurring at $r=0$, i.e. at the center of the nozzle. The maximum velocity $\left(v_{\mathrm{m}}\right)$ decreases with distance from the nozzle $x$ and can be estimated from (2) by measuring airflow rate $Q$ through the tube, since $v_{0}=Q / \pi r_{0}^{2}$. By measuring the position of the hair relative to the nozzle, in terms of $r$ and $x$, we can predict the local air velocity that hair is experiencing.

Finding an analytical prediction for the amplitude of hair vibration due to the local flow velocity is a trickier question, since the dynamics governing turbulent flows past cylinders are complex. In general it is understood that pressure variations in turbulent flows across cylinders can excite both resonant and non-resonant vibrations. For the latter case, the vibrational energy is distributed among the natural frequencies of the cylinder, as in a free vibration case [38]. Experimental data in air suggest that the root mean squared $\left(y_{r m s}\right)$ amplitude of flow induced vibration is proportional to $v^{3 / 2}[39,40]$. If the output power of the sensor $i_{r m s}^{2}$ is proportional to the vibrational power of the hair $y_{r m s}^{2}$, then

$$
y_{r m s}^{2} \propto i_{r m s}^{2}=\beta v^{3}
$$

where $\beta$ is an arbitrary scaling constant which we will refer to as the "sensitivity" of the sensor. This relationship holds as long as the flow is fast enough and away from a resonance condition. The conditions were well met for this study according to the criteria explained by Paidoussis [38] for flows above $3 \mathrm{~m} / \mathrm{s}$, hair diameters below 0.3 $\mathrm{mm}$ and resonances below $2 \mathrm{kHz}$.

\subsection{Bilayer Mechanoelectrical Transduction}

The droplet interface bilayer can be modelled in general as a capacitor and resistor in parallel $[41,42]$. However, large resistance values $(>10 \mathrm{M} \Omega)$ for well-formed bilayers result in primarily capacitive behavior between excitation frequencies of 1-10,000 $\mathrm{Hz}$ $[43,23]$. The hair vibrational frequencies observed in this study fall well inside this range, thus we consider the membrane as a simple capacitor. For a constant voltage applied to the bilayer, the trans-membrane current can be described as

$$
i=V \frac{\mathrm{d}}{\mathrm{dt}}(C(V, t))
$$


where $V, i$ and $C(V, t)$ represent voltage and current across the bilayer membrane and its capacitance, respectively. For high frequency oscillations $(>2 \mathrm{~Hz})$, the membrane will bend back and forth around some initial curvature[44]. The bending introduces a change in membrane area, which is directly related to membrane capacitance, hence the time dependent capacitance in (4). This phenomenon has been observed many times in literature and is referred to as mechanocapacitance [25, 26, 45, 44].

One must also take into account that membrane capacitance is a function of the applied voltage due to the electrowetting effect [46]. An applied electric field will reduce the contact angle between two aqueous volumes to produce a larger contact area. Due to this increase in area, it has been established experimentally [42, 41] that bilayer capacitance $C$ will vary with the square of the applied voltage $V$

$$
C(V, t)=C_{0}(t)\left(1+\alpha V^{2}\right),
$$

where $C_{0}$ is the capacitance at zero volts and $\alpha$ is the voltage dependence, which is sensitive to both lipid type and bilayer geometry. For a sinusoidally varying, zero-volt membrane capacitance

$$
C_{0}(t)=C_{0}^{\mathrm{m}} \sin (\omega t)+C_{0}^{\mathrm{i}},
$$

we obtain the output current by substituting (6) and (5) into (4):

$$
i(V, t)=\omega C_{0}^{\mathrm{m}} \cos (\omega t)\left[V+\alpha V^{3}\right] .
$$

Here, $C_{0}^{\mathrm{i}}$ and $C_{0}^{\mathrm{m}}$ represent the undisturbed zero-volt capacitance and the capacitance at a maximum amplitude of oscillation, respectively. The variable $\omega$ represents the oscillation frequency. Thus, mechanocapacitive current is proportional to voltage, stimulus frequency, amplitude change in capacitance and it lags membrane deflection by 90 degree phase[47]. Electrowetting effects give the bilayer membrane a cubic voltage sensitivity. The voltage sensitivity, however, is only an amplification factor for the membrane output at zero volts and should not, in theory, change its frequency output characteristics. It should also be noted that membrane oscillations are in general more complex than the simple model presented here. For example, flexoelectric effects $[26,44]$ have not been considered. Nevertheless, this model provides useful insight into the the behavior governing AHC current output.

\section{Results and discussion}

This study focuses on sensing spatial variations in airflow, a common function of many biological hair cells $[1,2,4,5]$. Arrays of three artificial hair cells map air velocity to location by measuring the individual excitation of each hair cell. The AHC outputs are combined into one channel, requiring a demultiplexing operation to recover individual hair cell information. This is achieved by tuning each AHC hair length to a unique frequency response and recovering individual sensor information based on the frequency content of the signal (TFRE method). This section first builds the foundation for TFRE by characterizing the response of single AHCs to airflow and comparing the results to theory. It then examines how repeatable the response is with respect to the AHC fabrication. Finally, it explains the TFRE methodology and applies it in combination with experimental data to interpret airflow information and assess AHC array sensing capabilities. 


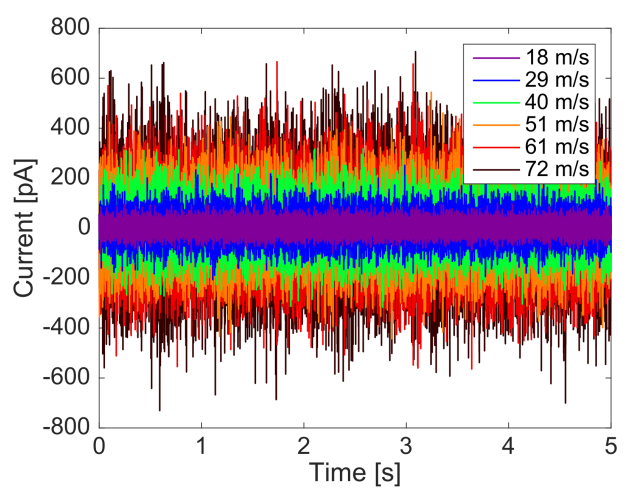

(a)

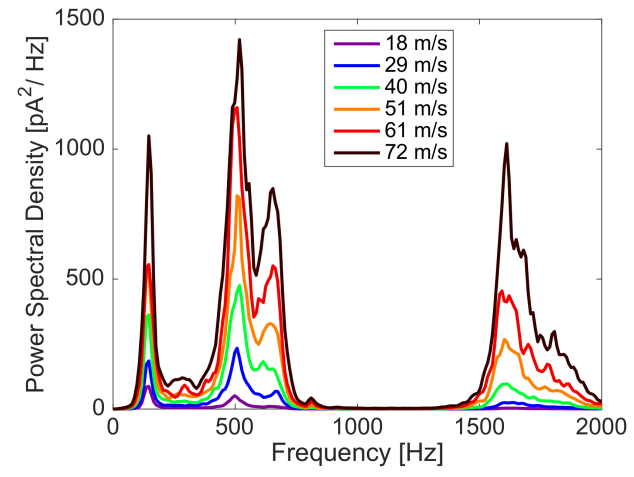

(b)

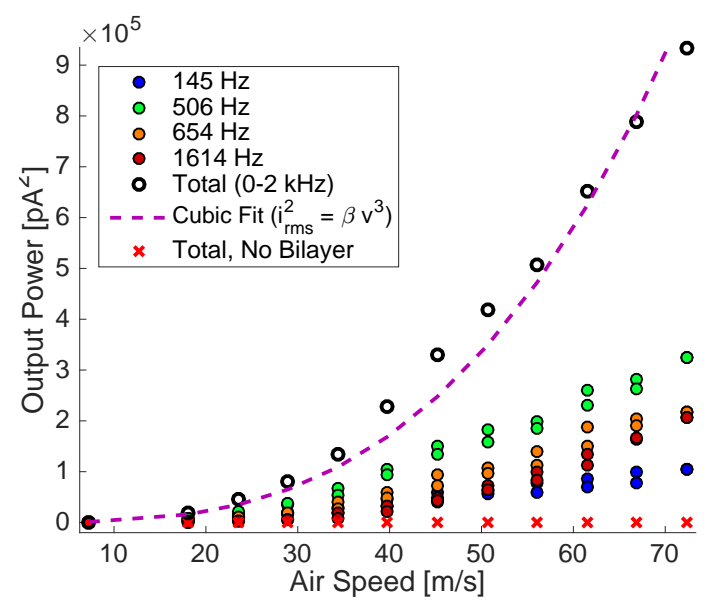

(c)

Figure 4: a) Measured current output for a single $\mathrm{AHC}$ at various airflow speeds. The hair length was $36 \mathrm{~mm}$ and excited by an air source $40 \mathrm{~mm}$ away. The membrane had $990 \mathrm{pF}$ capacitance with a $50 \mathrm{mV}$ bias potential applied. b) Power spectral density of the output currents in (a), computed using Welch's method. c) Output power (integrated PSD) versus flow speed for a $10 \mathrm{~Hz}$ band centered at the natural frequencies (colored dots) and total spectrum from $0-2 \mathrm{kHz}$ (white dots) from (b). Total output power cubic sensitivity to air speed: $\beta=2.6 \mathrm{pA}^{2} /(\mathrm{m} / \mathrm{s})^{3}$ with $R^{2}=0.98$.

\subsection{AHC response to airflow}

In previous studies, laminar flow has been shown to cause only a static deflection in the hair [48]. Turbulent airflows, however, excite the hair into continuous free vibration about a static deflection. Aside from an initial transient, the static deflection has no effect on the response of the bilayer; according to (4), the bilayer current output is dependent only on the rate of change of hair deflection. Output current measurements in figure $4 \mathrm{a}$ of a single $\mathrm{AHC}$ at various airflows $(0-72 \mathrm{~m} / \mathrm{s})$ show that output rms amplitude increases with speed. The frequency content of the signals is consistent, as seen in $4 \mathrm{~b}$. In fact, the measured peak frequencies varied less than $3 \%$ over 22 measurements. In addition, these frequencies agree with a spring-supported beam 
model of the AHC as well as direct laser vibrometry measurement of hair vibration under base excitation [33]. This supports the theory that the hairs are excited into free vibration as long as the flow velocity is far from exciting resonance (see section 3.1). The velocity only influences the magnitude of this vibration. Figure $4 \mathrm{c}$ shows how the output power (integrated PSD) increases with velocity. The output power is shown for the full $0-1.25 \mathrm{kHz}$ spectrum (white dots) as well as for $10 \mathrm{~Hz}$ integration bands around the natural frequencies (colored dots). The total power agrees well with the cubic relationship in (3), with a cubic sensitivity $\beta=2.6 \mathrm{pA}^{2} /(\mathrm{m} / \mathrm{s})^{3}$ for this experiment $\left(R^{2}=0.98\right)$. The frequency-specific output power did not follow the relationship quite as closely. High frequency power tended to increase disproportionately to low frequency power. Notice how in figure 4c, the power at $1614 \mathrm{~Hz}$ begins low but eventually overtakes the $145 \mathrm{~Hz}$ power. In general, the experimental value of $\beta$ was sensitive to membrane capacitance, hair length and hydrogel properties (e.g. size, composition and age). These results not only serve to characterize AHC behavior for the development of TFRE, but also support the idea that the AHC output current (via membrane transduction) does not significantly alter the dynamics of the mechanical signal (i.e. the hair).

The high aspect ratios of artificial hair cells allow for local velocity measurements. Figure 5 shows the averaged results of four different normalized trials using. The experiment used two different AHCs (37 and $26 \mathrm{~mm}$ hairs) with cubic sensitivities calculated as 6.02 and $9.35 \mathrm{pA}^{2} /(\mathrm{m} / \mathrm{s})^{3}$, respectively. The AHCs were tested by moving the air source in the same fashion as in figure 3 except with only one AHC connected at a time. Two different maximum velocities were used $(25.5 \mathrm{~m} / \mathrm{s}$ and 32 $\mathrm{m} / \mathrm{s})$. The estimates were calculated by converting the the total output power of the sensor to velocity using (3). As demonstrated by the figure, the estimates had high standard deviation (about $16 \%$ of the theoretical value). The average value is a "smoothed" version of the theoretical curve. While some of this discrepancy is due to fitting error in the sensitivity estimate, an additional error source was the air nozzle alignment. Because the air velocity profile has quite high gradients, especially around the center, slight variations in the air source position can result in measurable variations in the $\mathrm{AHC}$ excitation.

Finally, it is important to note that the hair-flow interaction studied herein is fundamentally different to a majority of previous AHC research [11, 19, 18, 13], which measured low fluid flows based on cilia deflection. As seen above, these AHCs rely on a vibrational response to fast, turbulent flows. As a result, the flow velocities encountered in this study are several orders of magnitude larger that those mentioned in the introduction section $(\mathrm{mm} / \mathrm{s}$ versus $\mathrm{m} / \mathrm{s})$. The slenderness and flexibility of the cilia allows them to operate in such a variety of regimes without overloading or interfering with the flow itself. The AHC design presented could be adapted to measure slow, laminar fluid flow, however, the bilayer vibrational response is of interest not only for flow but also for sensing acoustic and vibrational signals.

\subsection{Methodology and assumptions of tuned frequency response encoding}

When measuring with full array, the outputs of three AHCs are combined in parallel to a single channel, as show in figure 3 . According to Kirchoff's current law, the measured current is a sum of the individual unit output currents $\left(i_{1}+i_{2}+i_{3}\right)$ [49]. Each AHC hair length was tuned manually so that each frequency response had minimal overlap. The lengths of AHCs 1,2 and 3 were 33,26 and $20 \mathrm{~mm}$ respectively. In order to 


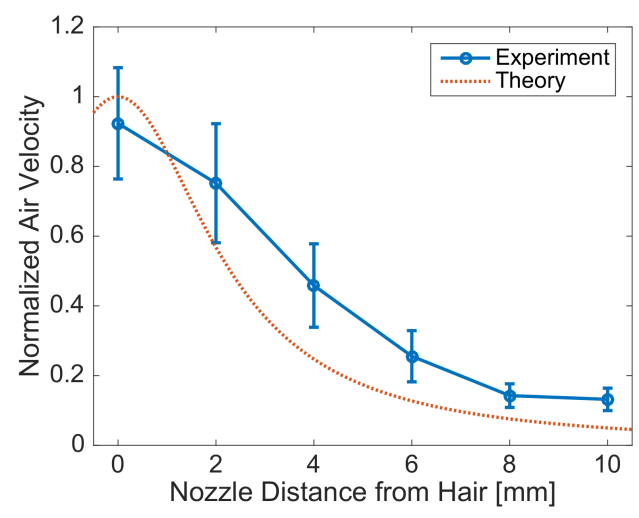

Figure 5: Experimentally measured velocity profile based the cubic sensitivity relationship (eq. (3)). Results are normalized to the experimental curve and then averaged over four tests: two velocities for two different AHCs. Error bars indicate one standard deviation.

recover the individual currents $i_{1}, i_{2}$, and $i_{3}$, we require some prior knowledge of each AHC frequency response. Furthermore, to estimate the velocity at each AHC, we require a sensitivity $\beta$ for the relationship in (3).

We characterize this behavior in a brief $(10$ minute $)$ pre-test phase by independently forming and exciting each $\mathrm{AHC}$. With only the $\mathrm{AHC}$ in question connected to the Axopatch DAQ, we first form the bilayer membrane, wait for it to stabilize, measure the membrane capacitance and the excite it at two velocities that span the testing range, 12 and $26 \mathrm{~m} / \mathrm{s}$. The two velocity points are enough to overdefine the sensitivity factor $\beta$ in (3), which depends on only one parameter. The velocities are correlated to the full spectrum power using a least squares fit, just as in figure 4c. Although more points provide a better estimate, it is also important to keep the pre-test brief in order to ensure consistent bilayer properties and stability.

The current response of each AHC is used as a reference on which to decompose the current content of the full array. Reference AHC responses from three separate tests are shown in figure 6a. Each test involved a new bilayer formation, but used the same AHCs (i.e same hair and hydrogel properties). The frequency peaks are very consistent across tests, with $1.3 \%$ average difference and $0.4 \%$ standard deviations. This consistency did not hold with different hydrogels or even hydrogels that had aged a few days. It is also clear from the figure that membrane capacitance dictates output power, and thus also sensitivity. Figure $6 \mathrm{~b}$ shows how the sensitivity of each AHC, calculated using the two velocity method, varies across five tests. In order to account for the effects of capacitance on sensitivity, the $\beta$ values are corrected to a nominal $300 \mathrm{pF}$ capacitance by dividing by $C_{\text {norm }}=C / 300$, where $\mathrm{C}$ is the measured membrane capacitance. In three tests (same as those from figure 6a), only the bilayers were changed (orange line). In the other two tests (not shown), both hydrogels and bilayers were different (blue line). Notice how in general, the sensitivity of the AHCs tends to decrease with hair length. However, the use of different hydrogels increases its variation. Overall, from the results in figure $6 \mathrm{a}$, we observe that frequency content depends primarily on the mechanical properties of the AHC (e.g hydrogel age, size 

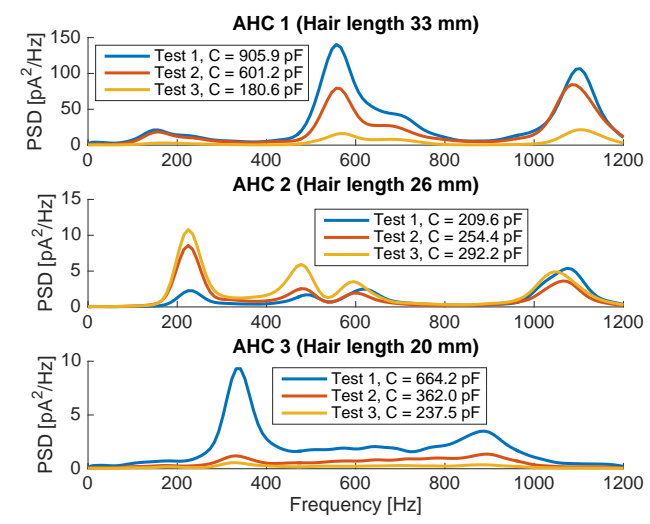

(a)

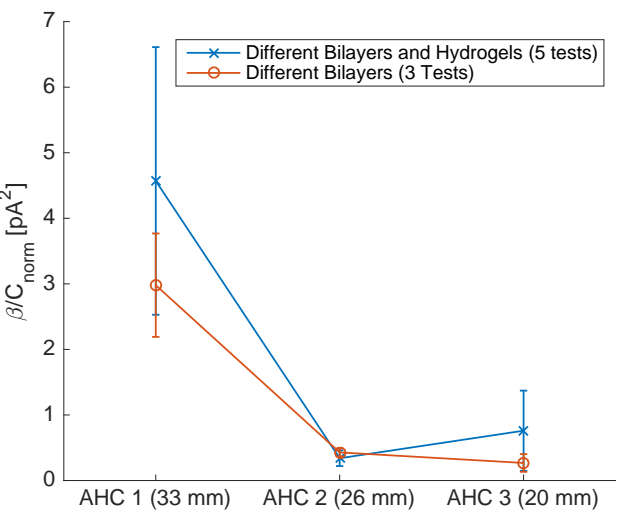

(b)

Figure 6: a) Reference AHC responses for the array in section 2.3 for three independent tests all at $12 \mathrm{~m} / \mathrm{s}$ excitation flow. Each test involved a new bilayer formation, but used the same AHCs (i.e same hair and hydrogel properties). b) Average sensitivity values for each $\mathrm{AHC}$ from (a) with one standard deviation bars.

For a fully parallel array output, the decoding phase of TFRE makes two assumptions about the AHC output behavior. The first is that, within the 20-25 minute experimental time-frame, the bilayers undergo negligible changes in area. In other words, each AHC bilayer capacitance is assumed to stay constant throughout testing. In this way the sensitivity estimate is independent of time. The assumption is based on the observation of stable undisturbed bilayer areas for over 25 minutes after formation, as shown in Appendix A. The second assumption, based on the experimental observations above, holds that the AHC out frequency content will not vary with excitation velocity. In addition, we assume that the power at frequency varies uniformly, although this is not always true experimentally. To help with this assumption, all signals are low-pass filtered at $900 \mathrm{~Hz}$ to remove large variations at high frequency modes. Note that figure 6 a shows the data prior to filtering.

Figure 3 illustrates how TFRE estimates the contribution of each AHC to the total array output. It uses functional approximations of the reference responses in figure $6 \mathrm{a}$ (red, blue and green curves) to represent the frequency content of each AHC. A nonlinear least squares algorithm [50,51] determines the appropriate scaling of each function $\left(\hat{c}_{1}, \hat{c}_{2}, \hat{c}_{3}\right)$. The estimated velocities are simply the power of the scaled functions times the inverse sensitivity $\left(\beta^{-1}\right)$ corresponding to each AHC, based on 3 . The power in this case is the integral of the PSD function and is equivalent to the square of the rms current $i_{r m s}^{2}$. See Appendix B and equation B.3 for more details on this process.

The method was partly inspired by the biological function of the basilar membrane, which performs a similar but inverse signal processing operation [1]. The basilar membrane is a flexible structure that stretches along the center of the cochlea. The stiffness varies gradually along its length such that the location of resonance is a function of the input frequency [9]. Thus, through an array of biological hair cells that line the membrane from end to end, the brain can map an excitation at a specific 


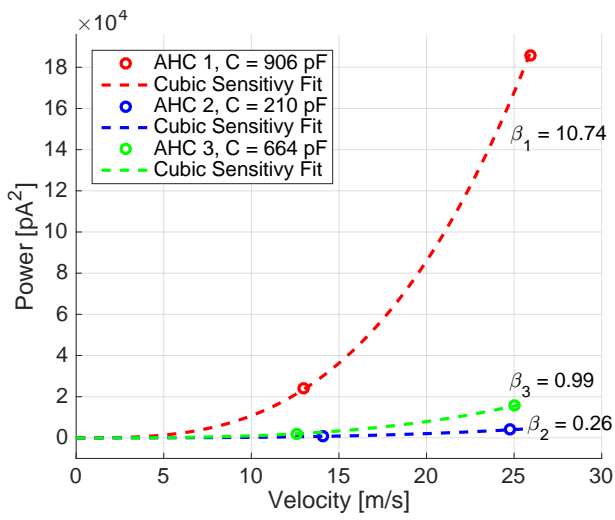

(a)

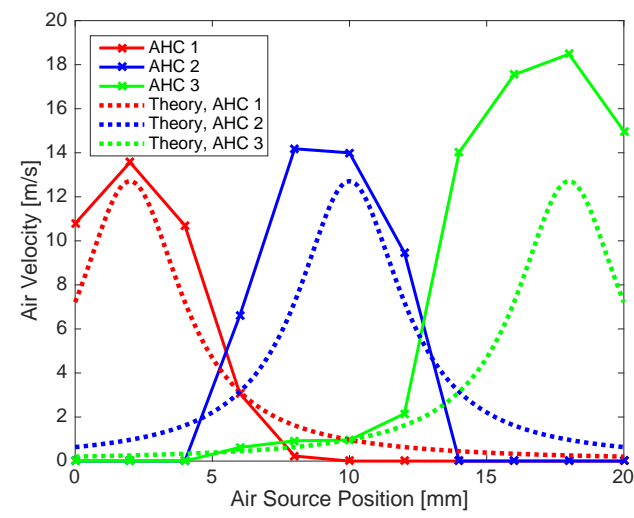

(b)

Figure 7: a) Pre-test sensitivity calculation of the three AHCs. The circular data points indicate the total spectrum power of the output current at two velocity points. The dashed line is the best fit of (3) to the points. b) TFRE velocity estimates (red, blue, and green curves) for each air source position (step size of $2 \mathrm{~mm}$ ). AHCs 1, 2 , and 3 were positioned at 2, 10 and $18 \mathrm{~mm}$, respectively. The theoretical velocity values are shown by the colored dotted lines.

location to a particular sound frequency. The arrays in this study, on the other hand, have a unique frequency response associated with each location. This maps a set of frequencies to a particular location. While TFRE cannot match the accuracy of multiple independent measurement channels, it is quite useful when these are limited. More generally, it demonstrates an approach to encoding information as frequency.

\subsection{Spatial flow measurements via TFRE}

Example experimental results of TFRE for a single sweep test are shown in figure 7 . The data correspond to test 1 in figure 6 a, where AHCs 1, 2, and 3 had measured capacitances of 906,210 , and $664 \mathrm{pF}$, respectively. Figure 7 a shows the sensitivity fits calculated for each AHC in the pre-test phase. The cubic fits showed strong agreement in all cases $\left(R^{2}>0.99\right)$. Notice how the sensitivity values $\beta$ vary widely in relation to each other. The solid lines in figure $7 \mathrm{~b}$ represent an estimate of velocity as seen by each AHC for the corresponding air source position on the horizontal axis. Ideally each should trace out the velocity profile described by (1), as indicated by the dotted lines. We observe that the estimated profile peaks align well with the peaks in the theoretical prediction. This indicates that the frequency content of the array output is clear and consistent enough for the decoding algorithm to reliably separate the component signals. This is a product of high signal to noise ratio and good signal isolation among AHCs. The magnitudes, however, are over-predicted in a similar manner to the single AHC tests (figure 5).

It was observed that in for some inaccurate predictions, the total output power of the AHC array was very different than the power observed in the pre-test phase for the same velocity. For example, as shown in figure 8a, when the air source was centered on AHC 3 during array testing so that it was excited with $12.7 \mathrm{~m} / \mathrm{s}$ airflow, the full array output power was $6015 \mathrm{pA}^{2}$. In the pre-test several minutes prior, the 


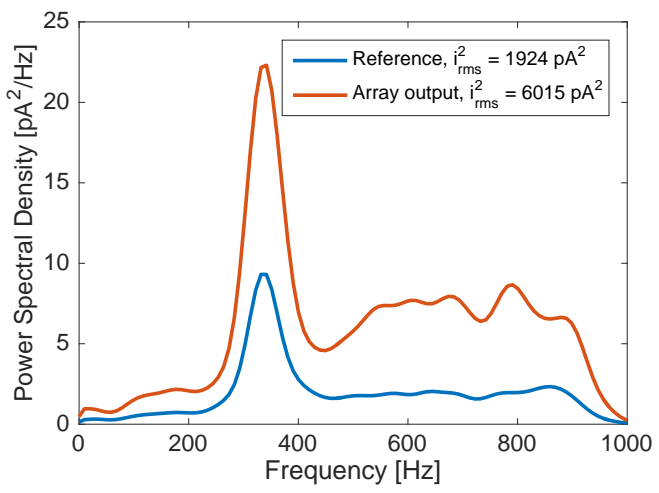

(a)

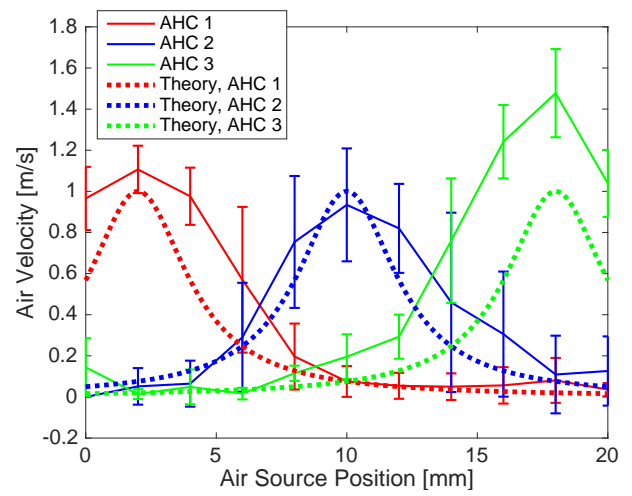

(b)

Figure 8: a) PSD of array output for airflow centered on AHC 3 (position $18 \mathrm{~mm}$ ) versus the reference measurement for the same AHC. Both curves were calculated for air flow at $12.6 \mathrm{~m} / \mathrm{s}$. b) Mean and standard deviation for three TFRE velocity estimates on the same set of AHCs with different bilayers. The measurement velocities are normalized to the maximum theoretical velocity.

AHC 3 power output for the same velocity was considerably lower, $1924 \mathrm{pA}^{2}$. Even though other AHC signals contribute to the array output, it is clear from the frequency content that this is due to an increase in the $\mathrm{AHC} 3$ response. The opposite effect was observed for under-prediction cases. This effect is most likely caused by time varying membrane capacitance, since all other factors that affect sensitivity (hair length, hydrogel properties) are constant. In some cases, AHC capacitance was seen to vary by as much as $70 \%$ from pre- to post- experiment, which supports this theory.

Figure $8 \mathrm{~b}$ shows the mean and standard deviations among three tests with the same AHCs but different bilayers. These tests are the same as 1,2, and 3 from figure 6. AHCs 1 and 3 consitently over predicted the theoretical curve. AHC 2 fluctuated between over- and under-prediction. Standard deviation was $15 \%$ on average but increase to about $30 \%$ at points between AHCs, i.e. where AHCs received equal excitation. The prediction variation is attributed to fluctuations in membrane sensitivity (above), errors in the sensitivity fitting process (figure 7a) and small variations in the nozzle alignment. Fitting errors can be reduced by sampling 5 or more points in the pre-test phase. Nozzle alignment issues could be eliminated by using dense arrays ( $\approx 1 \mathrm{~mm}$ spacing) and measuring a fixed flow profile.

\section{Conclusions}

Synthetic lipid bilayer membranes have show potential as mechanoelectrical transducers in AHC array applications. Their current output is reflective vibrational behavior of the hair without introducing undesired dynamics. For example, literature suggests that hair vibrational power has a proportional relationship to air velocity cubed, and the AHC current output power reflects this behavior. In addition, we have shown that the current output frequency content is independent of the bilayer size or the bilayer itself, only the mechanical properties of the AHC. This enables the development of a sensing strategy (tuned frequency response encoding) to map a 
spatially variable velocity flow profile using AHC arrays. Errors in the prediction of the magnitude of the profile were linked to fluctuations in the sensors' sensitivity to airflow. Bilayers, as an organic structures, are more variable than traditional mechanoelectrical components. Changes in bilayer capacitance are indistinguishable from changes in the airflow magnitude and thus reduce the accuracy of our estimation method. Although undisturbed bilayer membranes have demonstrated very stable capacitances for periods longer that those tested, it is possible that continuous excitation of these structures may undermine this stability.

The AHCs also demonstrated a general ability to withstand the dynamic fabrication and testing environment, which involved droplet manipulations on the order of millimeters, translation of the substrate on the order of centimeters, and hair vibrations with millimeter scale amplitudes. Theoretically, the array size could be increased indefinitely if three conditions are met: bilayers remain stable throughout assembly, electrical noise in the bilayer electrodes remains low and the cilia lengths are chosen so that each AHC response is differentiable by TFRE.

In design terms, we observe that the hairs provide measurements of a very narrow section of the flow field. This is a general advantage of hair cell sensors. We were able to spatially sample different points of the flow profile simultaneously by geometric arrangement of the AHC units. The array configuration tested here features $8 \mathrm{~mm}$ lateral resolution. Subsequent versions of this systems could increase this resolution to approximately $1-2 \mathrm{~mm}$ with minimal redesign, simply by reducing AHC spacing and hydrogel size. Furthermore, based on the principles established here, additional membrane-based AHC strategies may be developed. These could include two dimensional arrays, where the AHCs are staggered and/or placed in different orientations. It could also include more complex hydrogel/bilayer networks such as a long hydrogel with many hairs and one bilayer.

Finally, the results validate TFRE as well as the array sensing concept. The decoding algorithm showed good performance and robustness in separating spectral information into component signals, even in cases where the output deviated from the method's simplifying assumptions. Its effectiveness could be improved by measuring a broader frequency spectrum and optimizing the level of frequency separation. This will enable TFRE with more hairs and closer spacings. The method's hierarchical structure-a weighted fit built from reference responses-can be adapted to any frequency encoding application. Possibilities include more complex AHC sensing scenarios where one bilayer is used to measure multiple hair vibrations and general applications limited to few output channels.

\section{Acknowledgments}

The authors thank the generous financial support from the National Science Foundation (Grant CMMI-1129951).

\section{References}

[1] A. J. Hudspeth. How the ear's works work. Nature, 341:397-404, 1989.

[2] Sanjay P. Sane and Matthew J. McHenry. The biomechanics of sensory organs. In Sensory Biomechanics, pages i8-i23. Society for Integrative and Comparative Biology, Oxford University Press, 2009.

[3] Albert, Friedrich, Dechant, and et al. Arthropod touch reception: spider hair sensilla as rapid 
touch detectors. Journal of Comparative Physiology A: Neuroethology, Sensory, Neural, and Behavioral Physiology, 187(4):303-312, 2001.

[4] FG Barth. How to catch the wind: spider hairs specialized for sensing the movement of air. Naturwissenschaften, 87(2):51-58, 2000.

[5] J. Montgomery, S. Coombs, and M. Halstead. Biology of the mechanosensory lateral line in fishes. Reviews in Fish Biology and Fisheries, 5:399-416, 1995.

[6] James O Pickles and David P Corey. Mechanoelectrical transduction by hair cells. Trends in neurosciences, 15(7):254-259, 1992.

[7] T Teyke. Collision with and avoidance of obstacles by blind cave fishanoptichthys jordani (characidae). Journal of Comparative Physiology A, 157(6):837-843, 1985.

[8] Michael E McConney, Nannan Chen, David Lu, Huan A Hu, Sheryl Coombs, Chang Liu, and Vladimir V Tsukruk. Biologically inspired design of hydrogel-capped hair sensors for enhanced underwater flow detection. Soft Matter, 5(2):292-295, 2009.

[9] Ben M Clopton and Herbert F Voigt. The peripheral auditory system. 2006.

[10] Friedrich G Barth, Joseph AC Humphrey, and Timothy W Secomb. Sensors and sensing in biology and engineering. Springer, 2003.

[11] Chang Liu. Micromachined biomimetic artificial haircell sensors. Bioinspiration and Biomimetics, 2:S162-S169, 2007.

[12] Junliang Tao and Xiong Bill Yu. Hair flow sensors: from bio-inspiration to bio-mimickinga review. Smart Materials and Structures, 21(11):113001, 2012.

[13] G. J. Krijnen and et al. Mems based hair flow-sensors as model systems for acoustic percetion studies. Nanotechnology, 17:S84-S89, 2006.

[14] Jack Chen, Jonathan Engel, Nannan Chen, Saunvit Pandya, Sheryl Coombs, and Chang Liu. Artificial lateral line and hydrodynamic object tracking. In Micro Electro Mechanical Systems, 2006. MEMS 2006 Istanbul. 19th IEEE International Conference on, pages 694697. IEEE, 2006.

[15] Patrick D McGary, Liwen Tan, Jia Zou, Bethanie JH Stadler, Patrick R Downey, and Alison B Flatau. Magnetic nanowires for acoustic sensors. Journal of Applied Physics, 99(8):08B310, 2006.

[16] Xiong Yu, Junliang Tao, and Jim Berilla. A bio-inspired flow sensor. In SPIE Smart Structures and Materials + Nondestructive Evaluation and Health Monitoring, pages 764618-764618. International Society for Optics and Photonics, 2010.

[17] Ch Brücker, J Spatz, and W Schröder. Feasability study of wall shear stress imaging using microstructured surfaces with flexible micropillars. Experiments in fluids, 39(2):464-474, 2005.

[18] Nannan Chen, Craig Tucker, Jonathan M Engel, Yingchen Yang, Saunvit Pandya, and Chang Liu. Design and characterization of artificial haircell sensor for flow sensing with ultrahigh velocity and angular sensitivity. Microelectromechanical Systems, Journal of, 16(5):999-1014, 2007.

[19] Sergiy Pelechanko and et al. Hydrogel-encapsulated microfabricated haircells mimicking fish cupula neuromast. Advanced Materials, 19:2903-2909, 2007.

[20] RK Jaganatharaja, CM Bruinink, BM Hagedoorn, ML Kolster, TSJ Lammerink, RJ Wiegerink, and GJM Krijnen. Highly-sensitive, biomimetic hair sensor arrays for sensing lowfrequency air flows. In Solid-State Sensors, Actuators and Microsystems Conference, 2009. TRANSDUCERS 2009. International, pages 1541-1544. IEEE, 2009.

[21] Remco J Wiegerink, Arjan Floris, Ram K Jaganatharaja, Nima Izadi, Theo SJ Lammerink, and Gijs JM Krijnen. Biomimetic flow-sensor arrays based on the filiform hairs on the cerci of crickets. In Sensors, 2007 IEEE, pages 1073-1076. IEEE, 2007.

[22] Yingchen Yang, Jack Chen, Jonathan Engel, Saunvit Pandya, Nannan Chen, Craig Tucker, Sheryl Coombs, Douglas L. Jones, and Chang Liu. Distant touch hydrodynamic imaging with an artificial lateral line. Proceedings of the National Academy of Sciences, 103(50):1889118895, 2006.

[23] Stephen A Sarles and Donald J Leo. Membrane-based biomolecular smart materials. Smart materials and structures, 20(9):094018, 2011.

[24] Stephen A Sarles, John DW Madden, and Donald J Leo. Hair cell inspired mechanotransduction with a gel-supported, artificial lipid membrane. Soft Matter, 7(10):4644-4653, 2011.

[25] Alfred L Ochs and Robert M Burton. Electrical response to vibration of a lipid bilayer membrane. Biophysical journal, 14(6):473-489, 1974.

[26] AG Petrov and VS Sokolov. Curvature-electric effect in black lipid membranes. European Biophysics Journal, 13(3):139-155, 1986.

[27] Stephen A. Sarles, L. Justin Stiltner, Chris B. Williams, and Donald J. Leo. Bilayer 
formation netween lipid-encased hydrogels contained in solid substrates. Applied Materials and Interfaces, 2(12):36543663, 2011.

[28] Stephen A Sarles and Donald J Leo. Regulated attachment method for reconstituting lipid bilayers of prescribed size within flexible substrates. Analytical chemistry, 82(3):959-966, 2010.

[29] Nima Tamaddoni and Andy Sarles. Fabrication and characterization of a membrane based hair cell sensor that features soft hydrogel materials. In ASME 2012 Conference on Smart Materials, Adaptive Structures and Intelligent Systems, pages 565-571. American Society of Mechanical Engineers, 2012.

[30] Stephen A Sarles, Kevin L Garrison, Taylor T Young, and Donald J Leo. Formation and encapsulation of biomolecular arrays for developing arrays of membrane-based artificial hair cell sensors. In ASME 2011 Conference on Smart Materials, Adaptive Structures and Intelligent Systems, pages 663-671. American Society of Mechanical Engineers, 2011.

[31] K. Tanuj Sapra and Hagan Bayley. Lipid-coated hydrogel shapes a components of electrical circuits and mechanical devices. Nature, 2:1-9, 2012.

[32] Nima Tamaddoni and Stephen A Sarles. Mechanotransduction of multi-hair droplet arrays. In ASME 2014 Conference on Smart Materials, Adaptive Structures and Intelligent Systems, pages V002T06A008-V002T06A008. American Society of Mechanical Engineers, 2014.

[33] Rodrigo Sarlo, Donald Leo, and Pablo Tarazaga. Spectral analysis and characterization of a membrane-based artificial hair cell sensor. In ASME 2014 Conference on Smart Materials, Adaptive Structures and Intelligent Systems, pages V002T06A010-V002T06A010. American Society of Mechanical Engineers, 2014.

[34] Joseph S Najem, Myles D Dunlap, Anthony Yasmann, Eric C Freeman, John W Grant, Sergei Sukharev, and Donald J Leo. Multifunctional, micropipette-based method for incorporation and stimulation of bacterial mechanosensitive ion channels in droplet interface bilayers. Journal of visualized experiments: JoVE, (105), 2015.

[35] William L Hwang, Min Chen, Brid Cronin, Matthew A Holden, and Hagan Bayley. Asymmetric droplet interface bilayers. Journal of the American Chemical Society, 130(18):5878-5879, 2008.

[36] Nima Tamaddoni, Eric C Freeman, and Stephen A Sarles. Sensitivity and directionality of lipid bilayer mechanotransduction studied using a revised, highly durable membrane-based hair cell sensor. Smart Materials and Structures, 24(6):065014, 2015.

[37] Nallamuthu Rajaratnam. Turbulent Jets. Elsevier, 1976.

[38] MP Paidoussis. Fluidelastic vibration of cylinder arrays in axial and cross flow: state of the art. Journal of Sound and Vibration, 76(3):333-335, 1981.

[39] RJ Gibert, J Chabrerie, and M Sagner. Vibrations of tube arrays in transversal flow. 1977.

[40] JB Nicolet, M Sagner, and G Regis. Vibrations de faisceaux de tubes sous excitation aerodynamique. Revue Générale de Thermique, 128:869-891, 1976.

[41] Stephen H White and TE Thompson. Capacitance, area, and thickness variations in thin lipid films. Biochimica et Biophysica Acta (BBA)-Biomembranes, 323(1):7-22, 1973.

[42] O Alvarez and R Latorre. Voltage-dependent capacitance in lipid bilayers made from monolayers. Biophysical journal, 21(1):1, 1978.

[43] Winfried Römer and Claudia Steinem. Impedance analysis and single-channel recordings on nano-black lipid membranes based on porous alumina. Biophysical journal, 86(2):955-965, 2004.

[44] EC Freeman, JS Najem, S Sukharev, MK Philen, and DJ Leo. The mechanoelectrical response of droplet interface bilayer membranes. Soft matter, 12(12):3021-3031, 2016.

[45] Alexander G Petrov. Planar Lipid Bilayers (BLM's) and Their Applications. Elsevier, 2003.

[46] Srikoundinya Punnamaraju and Andrew J Steckl. Voltage control of droplet interface bilayer lipid membrane dimensions. Langmuir, 27(2):618-626, 2010.

[47] Tibor Hianik and Victor Ivanovich Passechnik. Bilayer lipid membranes. Structure and mechanical properties. Springer, 1995.

[48] Jeffrey P Travis, Myles D Dunlap, Donald J Leo, and J Wallace Grant. Dynamic characterization of biomimetic artificial hair cells. In ASME 2013 Conference on Smart Materials, Adaptive Structures and Intelligent Systems, pages V002T06A011-V002T06A011. American Society of Mechanical Engineers, 2013.

[49] Paul Scherz, Simon Monk, Michael Margolis, and Chris Fitzer. Practical Electronics for Inventors. McGraw-Hill, 2013.

[50] TF Coleman and Y Li. On the convergence of reflective newton methods for large-scale nonlinear minimization subject to bounds. Mathematical Programming, 67(2):189-224, 1994.

[51] TF Coleman and Y Li. An interior, trust region approach for nonlinear minimization subject 
to bounds. SIAM Journal on Optimization, 6:418-445, 1994.

\section{Appendix A. Undisturbed Bilayer Area Stability}

DIBs formed using the techniques outilined in section 2.1 show stable area over time when undisturbed, i.e. no excitation. The bilayer area is directly linked to its capacitance. Sample experimental measurement on two bilayer of different size are show in figure A1.

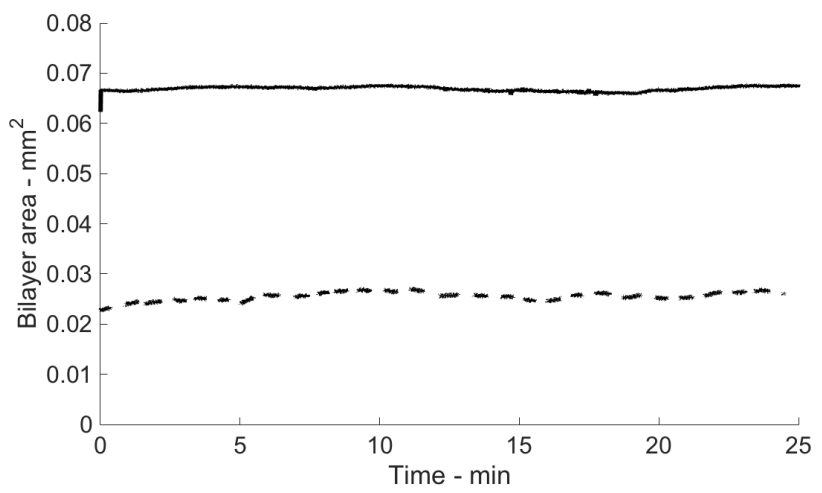

Figure A1: Experimentally measured area over time for two different bilayers.

\section{Appendix B. Tuned frequency response encoding algorithm}

Tuned Frequency Response Encoding (TFRE) algorithm is a hierarchical process with three building blocks:

(i) The reference response, a functional description of the frequency content of a single AHC. For best performance, each reference response must be as unique as possible, that is, the natural frequencies should be well separated.

(ii) The combined response, a weighted sum of reference responses. The weights are chosen to minimize the error between the combined response and a measured response

(iii) The sensitivity curve, a conversion from the power of a weighted response to excitation velocity.

The reference response is obtained from the power spectral density (PSD) of a decoupled AHC output to an constant airflow of arbitrary velocity. In this study, each reference response is calculated with the AHC independently connected and with airflow rates at 10 and $20 \mathrm{~L} / \mathrm{min}$ (corresponding approximately to 12 and 26 $\mathrm{m} / \mathrm{s}$ at the hair, respectively) with the air nozzle centered on the hair, and $-20 \mathrm{mV}$ applied voltage. A five second sample of the vibrational response is converted to a power spectral density (PSD). These PSDs serve as the "basis" functions on which to approximate the contribution of each AHC to the total output. 


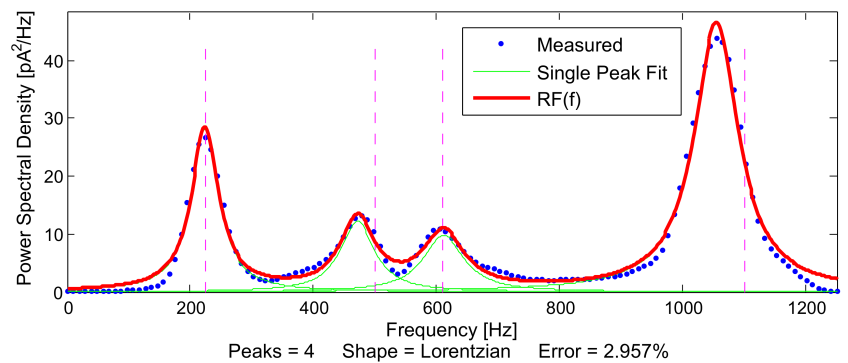

Figure B1: Basis response peak fit for a sample power spectral density of an individual AHC. Blue dots are the experimentally measured PSD values of 5-10 second time signal. The four green lines are the individual peak fits described by a single summation term in B.1. The red line is the sum of the four green lines, i.e. $R_{h}(f)$.

A fourth order peak fit of the experimental basis PSD provides a functional description of the individual spectral response of each AHC. An example fit is shown in figure $\mathrm{B} 1$. This reference response $R_{h}(f)$ is described as a sum of Lorentzian functions

$$
R_{h}(f)=\sum_{n=1}^{N} \frac{A_{n}}{\left[1+\left(\frac{f-f_{n}}{w_{n}}\right)^{2}\right]} .
$$

Each summation term describes a single peak of the output response. $N$ represents the fit order (total number of peaks). The independent variable $f$ is frequency, while subscripts $h$ and $n$ denote the AHC number and peak number respectively. $A_{n}$ is the peak amplitude, $f_{n}$ is the peak frequency and $w_{n}$ is the peak's half-width at half-height. The Lorentzian functional form was chosen as an adequate mathematical description of the modal peak shapes, with the added advantage that the parameters have intuitive physical interpretations. Other types of peak-shaped functions, such as Gaussian distributions, can also be used effectively.

Based on the assumption of uniform frequency content with respect to air velocity (see section 4.2), we expect a scalar multiple of the reference response to adequately represent all possible outputs for an AHC unit. Thus, the next step is to evaluate what combination of the three basis responses $\left(R_{1}(f), R_{2}(f), R_{3}(f)\right)$ best explains the total tuned frequency encoded array output, as shown previously in figure 3 . The combined response is expressed as

$$
C(\mathbf{c}, f)=\sum_{h=1}^{H} c_{h} R_{h}(f) .
$$

It is a sum of the unique basis responses $R_{h}$, each weighted by a coefficient $c_{h}$. The coefficient vector $\mathbf{c}=\left[\begin{array}{lll}c_{1} & c_{2} & c_{3}\end{array}\right]$ is initially unknown, but we use an iterative nonlinear fitting algorithm $[50,51]$ to find the values that minimize the least squares error between $C(\mathbf{c}, f)$ and the measured frequency response. The fitted coefficients $\hat{c}_{1}, \hat{c}_{2}, \hat{c}_{3}$ represent the fraction of excitation an AHC is receiving, relative to the reference PSD curve. These coefficients must be converted to a velocity estimate $\hat{v}_{h}$. This is done 
${ }_{539}$ using the sensitivity calculated in the pre-test phase. The velocity estimate for AHC $540 \quad h$ is

541

$$
\hat{v}_{h}=\left(\beta_{h}^{-1} \int_{f_{\min }}^{f_{\max }} c_{h} R_{h}(f) \mathrm{df}\right)^{\frac{1}{3}}
$$

${ }_{542}$ were $\beta_{h}$ is the AHC's cubic sensitivity to air velocity and $f_{\min }$ to $f_{\max }$ is the frequency ${ }_{543}$ band on which $\beta_{h}$ was calculated. The integral is analogous to the signal power. 